\title{
Stress Measurements in Alumina by Optical Fluorescence: Revisited
}

\author{
Robert F. Cook and Chris A. Michaels \\ National Institute of Standards and Technology, \\ Gaithersburg, MD 20899, USA
}

robert.cook@nist.gov

chris.michaels@nist.gov

\begin{abstract}
Stress measurements in single-crystal and polycrystalline alumina are revisited using a recently developed optical fluorescence energy shift method. The method simultaneously utilizes the $\mathrm{R}_{1}$ and $\mathrm{R}_{2} \mathrm{Cr}$-related ruby line shifts in alumina to determine two components of the stress tensor in crystallographic coordinates, independent of the intended or assumed stress state. Measurements from a range of experimental conditions, including high-pressure, shock, quasi-static, and bulk polycrystals containing thermal expansion anisotropy effects, are analyzed. In many cases, the new analysis suggests stress states and stress magnitudes significantly different from those inferred previously, particularly for shock experiments. An implication is that atomistic models relating stress state to fluorescence shift require significant refinement for use in materials-based residual stress distribution analyses. Conversely, the earliest measurements of fluorescence in polycrystalline alumina are shown to be consistent with recent detailed measurements of stress equilibrium and dispersion.
\end{abstract}

Key words: alumina $\left(\mathrm{Al}_{2} \mathrm{O}_{3}\right)$; fluorescence; pressure; shear; stress.

Accepted: July 17, 2019

Published: August 27, 2019

https://doi.org/10.6028/jres.124.020

\section{Introduction}

Alumina $\left(\mathrm{Al}_{2} \mathrm{O}_{3}\right)$ has long been used as a study material in considerations of microstructural residual stress distributions in polycrystals [1-3]. The equilibrium elastic constants of the anisotropic single-crystal $\mathrm{Al}_{2} \mathrm{O}_{3}$ (corundum) grains comprising a polycrystalline $\mathrm{Al}_{2} \mathrm{O}_{3}$ microstructure are well known [4], as are the anisotropic coefficient of thermal expansion (CTE) values for corundum [5]. The relationships between bulk polycrystal and constituent single-crystal elastic and thermal properties are well established [6], and it is well known that anisotropic single-crystal thermal expansion is ultimately responsible for residual stress development on cooling during polycrystal material fabrication. In addition, kinetic parameters such as diffusivity and creep susceptibility that characterize nonequilibrium mass motions that relax residual stresses, are also well known [2, 6]. As a consequence, stress development as a function of time, temperature, grain size, and grain-size distribution has frequently been modeled using these constants, coefficients, and parameters [1-3, 7-14]. A significant model motivation has been description of fracture properties of polycrystalline $\mathrm{Al}_{2} \mathrm{O}_{3}$ as influenced by residual stress, particularly the toughening by crack bridging of moderate-grain-size (about $20 \mu \mathrm{m}$ ) material [15] and the strength degradation by spontaneous microcracking of large-grain-size (about $100 \mu \mathrm{m}$ ) material [16-18]. The ability to sinter dense polycrystalline $\mathrm{Al}_{2} \mathrm{O}_{3}$ microstructures and fabricate materials with controlled grain sizes has enabled many investigations of these and other fracture phenomena. However, only rarely have the models been assessed by direct measurement of residual stress [19-23], impeding the goal of optimizing and predicting the fracture properties of $\mathrm{Al}_{2} \mathrm{O}_{3}$ and other polycrystalline ceramics by microstructural control. To enable better assessment and calibration of residual stress models, the study here used recent analyses and calibrations 
$[24,25]$ to revisit an extensive set of previous optical fluorescence-based stress measurements in singlecrystal and polycrystalline $\mathrm{Al}_{2} \mathrm{O}_{3}$. Revisiting the previous experiments using recent analyses will also increase confidence in the overall fluorescence-based stress measurement methodology.

The National Institute of Standards and Technology (NIST, formerly known as the National Bureau of Standards, NBS) has contributed significantly to the development of stress measurements in $\mathrm{Al}_{2} \mathrm{O}_{3}$ using optical fluorescence. The work here extends that contribution. Early work on related mechanical properties, conducted by Wachtman and colleagues at NBS in the 1960s, determined the fundamental elastic constants [4] and CTE values [5] of corundum single crystals. Later, Munro et al. at NIST in the 1990s critically evaluated a broad range of polycrystalline $\mathrm{Al}_{2} \mathrm{O}_{3}$ properties in the context of these constituent single-crystal properties [6]. The two most critical contributions to stress measurements in $\mathrm{Al}_{2} \mathrm{O}_{3}$ using fluorescence were made at NBS in the 1970s. In the early 1970s, Piermarini, Block, and colleagues at NBS established the ruby fluorescence scale for high-pressure measurements in diamond anvil cell (DAC) experiments [26]. Ruby is Cr-doped $\mathrm{Al}_{2} \mathrm{O}_{3}$ (see below), and legend has it that Wachtman originally suggested to Piermarini over lunch in the NBS cafeteria that perhaps pressure-induced fluorescence shifts in ruby could be used to calibrate DAC measurements. The ruby scale is now used extensively [27] in all high-pressure measurements. In the late 1970s, Grabner at NBS extended fluorescence shift measurements from scalar pressure effects to tensor stress effects [28]. The measurements and associated analysis thus became applicable to polycrystalline $\mathrm{Al}_{2} \mathrm{O}_{3}$. The tensor analysis was applied in many studies of bulk and thin-film polycrystalline $\mathrm{Al}_{2} \mathrm{O}_{3}$ materials, particularly by Clarke and colleagues in the 1990s, e.g., Ref. [29] (see Ref. [25] for a review of the diversity of applications).

A recent series of works initiated by NIST in the 2010s has demonstrated the advanced ability of hyperspectral optical fluorescence techniques to measure and map stress distributions in polycrystalline $\mathrm{Al}_{2} \mathrm{O}_{3}$ [24, 25, 30-33]. Spatial resolutions of $1 \mu \mathrm{m}$ over fields about $200 \mu \mathrm{m}$ square with stress resolutions of $10 \mathrm{MPa}$ were demonstrated, greatly extending earlier work [34] (see Ref. [24] for a review of fluorescence-based mapping). The basis of the technique is the shift in energies of two Cr-related fluorescence lines, $\mathrm{R}_{1}$ and $\mathrm{R}_{2}$, from their stress-free states (after correcting for temperature and composition effects) [25]. Cr is a ubiquitous substitutional impurity for $\mathrm{Al}$ in $\mathrm{Al}_{2} \mathrm{O}_{3}$, giving rise to optical absorption by electronic excitation in the blue-green region of the spectrum and hence the distinctive red color of ruby in transmitted light. Electronic relaxation leads to fluorescent photon emission of the doublet $R_{1}$ and $R_{2}$, which together are known as the ruby lines. These lines occur (coincidentally) in the red spectrum at wavelengths of $\lambda_{0}^{(1)}=694.3 \mathrm{~nm}$ and $\lambda_{0}^{(2)}=692.9 \mathrm{~nm}$, where the subscript 0 indicates a stress-free state, and the superscript (1) or (2) describes $\mathrm{R}_{1}$ or $\mathrm{R}_{2}$, respectively. The photon energies are usually quoted in wavenumbers, $1 / \lambda$, such that the stress-free energies are $v_{0}^{(1)}=14402.5 \mathrm{~cm}^{-1}$ and $v_{0}^{(2)}=14432.1 \mathrm{~cm}^{-1}$, omitting terms in Planck's constant, $h$, and the speed of light, $c$.

The recent works are based on the earlier development of ruby line shift measurement as a pressure gauge in DAC experiments [26]. The shift, $\Delta v^{(i)}=v^{(i)}-v_{0}^{(i)}$, where $v^{(i)}$ is the photon energy, is described by the scalar relation

$$
\Delta v^{(i)}=-\Pi_{p}^{(i)} p,
$$

where $p$ is the pressure, $\Pi_{p}^{(i)}$ are the piezospectroscopic pressure coefficients, and $i=1$ or 2 . For ruby, $\Pi_{p}^{(1)}=\Pi_{p}^{(2)}=7.60 \mathrm{~cm}^{-1} / \mathrm{GPa}$; i.e., the two lines shift equally under ideal hydrostatic pressure [25]. Under nonhydrostatic conditions, the two lines shift unequally, and a full tensor relation is required to relate the shifts to the components of the stress tensor [28]. Historically, however, usually only a single shift (often $\Delta v^{(1)}$ ) has been measured and related to a single stress component in the scalar manner of Eq. (1). The remaining stress components have been determined by assumed geometrical constraints, e.g., random grain orientation distribution in a polycrystal [29].

An innovation of the recent work at NIST [24] was the development and application of analysis for simultaneous measurement of both energy shifts, $\Delta v^{(1)}$ and $\Delta v^{(2)}$, to determine two independent components of the stress tensor. The recent work has also shown that earlier stress estimates may be in error but can be improved significantly by the use of simultaneous shift measurements [32]. This study revisited prior works that reported sufficient fluorescence information for analysis but did not report 
independent measures of stress, comparing the inferred stress (where reported) with that from application of the recent analysis. In most cases, explicit values of $\Delta v^{(1)}$ and $\Delta v^{(2)}$ were not reported, requiring preanalysis (e.g., reported changes in wavelength, $\Delta \lambda$, or relative wavenumber, $\Delta v^{(1)}-\Delta v^{(2)}$, were converted as specified below; pre-analysis uncertainty was negligible). In order to provide a broad context for measurement assessment, an extensive range of $\mathrm{Al}_{2} \mathrm{O}_{3}$ materials was considered. Both single-crystal and polycrystal data were analyzed, proceeding from high to low stress, including quasi-static high-pressure DAC loading [Fig. 1(a)], dynamic shock and quasi-static uniaxial loading [Fig. 1(b)], static thin film-onsubstrate biaxial loading [Fig. 1(c)], and polycrystalline triaxial residual stress [Fig. 1(d) and (e)]. In fact, all known relevant data were analyzed. To avoid circularity, previous works used in calibration of piezospectroscopic coefficients [25] were not included. The recent analysis is first summarized, followed by application of the analysis and fundamental coefficients established by NIST [25] to obtain new results from previous observations [28, 32, 35-44]. Discussion centers on the implications of the results for stress measurement and modeling.

\section{Analysis}

The change in energy with stress of the fluorescence lines in single-crystal $\mathrm{Al}_{2} \mathrm{O}_{3}$ is given by

$$
\Delta v^{(i)}=\Pi_{a}^{(i)} \sigma_{11}+\Pi_{a}^{(i)} \sigma_{22}+\Pi_{c}^{(i)} \sigma_{33},
$$

where the tensor axes $(1,2,3)$ correspond to the corundum crystallographic axes $(a, m, c)$, and $\Pi_{a}^{(i)}$ and $\Pi_{c}^{(i)}$ are the two nonzero piezospectroscopic coefficients for each line, giving four coefficients in all, calibrated as: $\Pi_{a}^{(1)}=2.98 \mathrm{~cm}^{-1} / \mathrm{GPa}, \Pi_{c}^{(1)}=1.64 \mathrm{~cm}^{-1} / \mathrm{GPa}, \Pi_{a}^{(2)}=2.64 \mathrm{~cm}^{-1} / \mathrm{GPa}$, and $\Pi_{c}^{(2)}=2.32 \mathrm{~cm}^{-1} / \mathrm{GPa}$ [25]. Equation (2) can be rewritten in two ways, first by collecting stress terms to extend Eq. (1) as

$$
\Delta v^{(i)}=\Pi_{\mathrm{M}}^{(i)} \sigma_{\mathrm{M}}+\Pi_{\mathrm{S}}^{(i)} \sigma_{\mathrm{S}}
$$

where

$$
\sigma_{\mathrm{M}}=\left(\sigma_{11}+\sigma_{22}+\sigma_{33}\right) / 3
$$

is a mean or spherical stress, and

$$
\sigma_{\mathrm{S}}=\left(2 \sigma_{33}-\sigma_{11}-\sigma_{22}\right) / 3
$$

is a shear or deviatoric stress. The effective piezospectroscopic coefficients are

$$
\Pi_{\mathrm{M}}^{(i)}=2 \Pi_{a}^{(i)}+\Pi_{c}^{(i)}
$$

and

$$
\Pi_{\mathrm{S}}^{(i)}=\Pi_{c}^{(i)}-\Pi_{a}^{(i)}
$$

For hydrostatic loading, as in Fig. 1(a), $\sigma_{\mathrm{S}}=0$, and $\sigma_{\mathrm{M}}=-p$, and thus $\Pi_{p}^{(i)}=\Pi_{\mathrm{M}}^{(i)}=2 \Pi_{a}^{(i)}+\Pi_{c}^{(i)}$, and, as the values of $\Pi_{p}^{(i)}$ are well known, this places constraints on the values of $\Pi_{a}^{(i)}$ and $\Pi_{c}^{(i)}$ [25]. Under general loading, e.g., as in Fig. 1(b) to (d), the energy shift for both lines is given by expanding Eq. (3) in matrix form as

$$
\left[\begin{array}{c}
\Delta v^{(1)} \\
\Delta v^{(2)}
\end{array}\right]=\left[\begin{array}{ll}
\Pi_{\mathrm{M}}^{(1)} & \Pi_{\mathrm{S}}^{(1)} \\
\Pi_{\mathrm{M}}^{(2)} & \Pi_{\mathrm{S}}^{(2)}
\end{array}\right]\left[\begin{array}{l}
\sigma_{\mathrm{M}} \\
\sigma_{\mathrm{S}}
\end{array}\right]
$$


(a) Hydrostatic

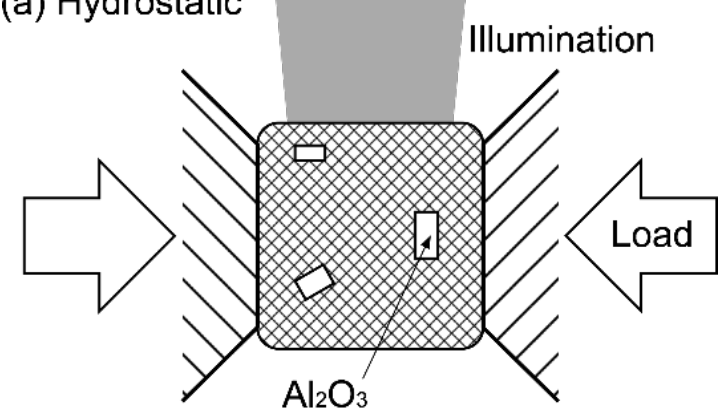

(b) Uniaxial

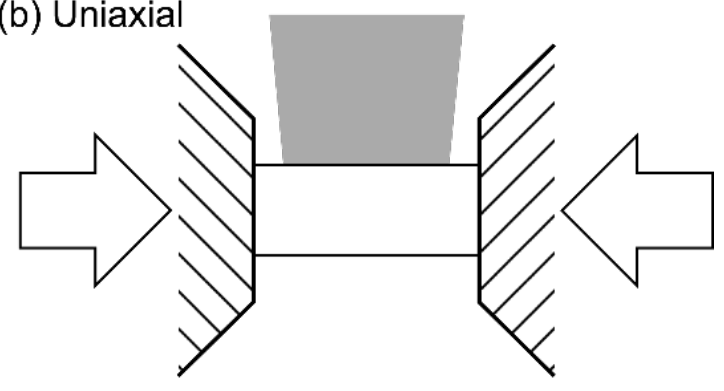

(c) Substrate

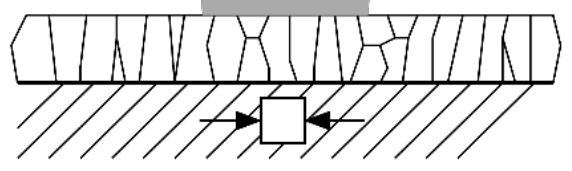

(d) Polycrystal

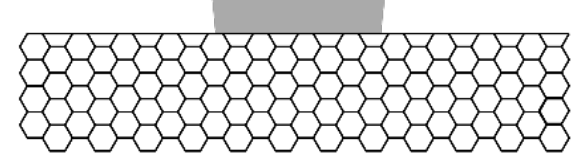

(e) Map

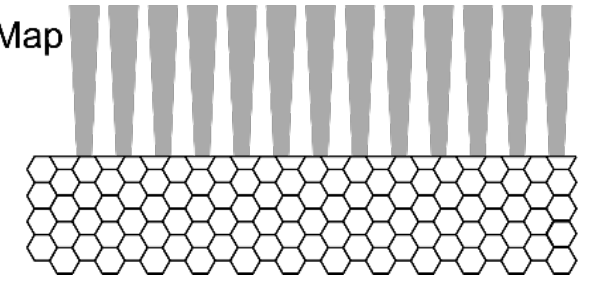

Fig. 1. Schematic diagrams of fluorescence-based techniques for determining stress in $\mathrm{Al}_{2} \mathrm{O}_{3}$. The illumination is shown as shaded bands in each diagram. (a) Hydrostatic loading generated by a confining medium (hatched) surrounding small crystals in a DAC. (b) Uniaxial loading of an oriented single crystal between two platens. (c) Biaxial loading generated on cooling by a thermal expansionmismatched substrate of an aligned polycrystalline film ( $a$ axis parallel to substrate). (d) Triaxial loading generated on cooling of a bulk polycrystal with randomly oriented grains, using integrated scan illumination by a single broad probe. (e) Polycrystal as in (d) with two-dimensional mapping using an array of repeated probes. 
Inverting Eq. (6) gives the two stress components as a function of the two measured shifts:

$$
\left[\begin{array}{l}
\sigma_{\mathrm{M}} \\
\sigma_{\mathrm{S}}
\end{array}\right]=\left(1 /\left(\Pi_{\mathrm{M}}^{(1)} \Pi_{\mathrm{S}}^{(2)}-\Pi_{\mathrm{S}}^{(1)} \Pi_{\mathrm{M}}^{(2)}\right)\right)\left[\begin{array}{cc}
\Pi_{\mathrm{S}}^{(2)} & -\Pi_{\mathrm{S}}^{(1)} \\
-\Pi_{\mathrm{M}}^{(2)} & \Pi_{\mathrm{M}}^{(1)}
\end{array}\right]\left[\begin{array}{l}
\Delta v^{(1)} \\
\Delta v^{(2)}
\end{array}\right] .
$$

Equation (7) applies both locally, where the stresses and shifts take their local measured values, and globally, where the stresses and shifts take global average values.

Alternatively, Eq. (2) may be rewritten by collecting crystallographic terms, as

$$
\Delta v^{(i)}=2 \Pi_{a}^{(i)} \sigma_{a}+\Pi_{c}^{(i)} \sigma_{c}
$$

where $\sigma_{a}$ and $\sigma_{c}$ are global average crystallographic stresses, and thus $\Delta v^{(i)}$ are taken here as global average shift values. Equation (8) may be expanded and inverted to give

$$
\left[\begin{array}{l}
\sigma_{a} \\
\sigma_{c}
\end{array}\right]=\left(1 /\left(2 \Pi_{a}^{(1)} \Pi_{c}^{(2)}-2 \Pi_{a}^{(2)} \Pi_{c}^{(1)}\right)\right)\left[\begin{array}{cc}
\Pi_{c}^{(2)} & -\Pi_{c}^{(1)} \\
-2 \Pi_{a}^{(2)} & 2 \Pi_{a}^{(1)}
\end{array}\right]\left[\begin{array}{c}
\Delta v^{(1)} \\
\Delta v^{(2)}
\end{array}\right],
$$

which gives two global stress components as a function of two global shifts. Equation (9) provides an approximation of the local field if $\sigma_{a}$ and $\sigma_{c}$ are the dominant local stress components. Both Eqs. (7) and (9) are independent of the orientation of the crystallographic $a$ - and $c$-axes relative to a laboratory or applied loading reference frame and hence provide a determination of the actual stress state from simultaneous measurements of $\Delta v^{(1)}$ and $\Delta v^{(2)}$, independent of any intended or achieved loading geometry.

\section{Observations}

\subsection{Single Crystals}

Figure 2 shows a color-filled contour map of the variation of $\sigma_{\mathrm{M}}$ as a function of $\Delta v^{(1)}$ and $\Delta v^{(2)}$ from Eq. (7); the contours are straight as the analysis is linear. The placement of a measured $\left[\Delta v^{(1)}, \Delta v^{(2)}\right]$ pair on this map provides the experimental value of $\sigma_{\mathrm{M}}$ via the contours. The behavior during two sets of singlecrystal high-pressure DAC experiments [Fig. 1(a)] by Okai et al. [35] is indicated by the symbols. The energy shift values were determined by converting the reported wavelength measurements (no corrections for temperature fluctuations were made here as the effect was expected to be small [25]). Closed symbols represent nominally hydrostatic experiments. Open symbols represent experiments in which the DAC confining media were known to lead to deviations from hydrostatic conditions. Initially, $\Delta v^{(1)}=\Delta v^{(2)}=0$ as $p=-\sigma_{\mathrm{M}}=0$. As $p$ increased, both $\Delta v^{(1)}$ and $\Delta v^{(2)}$ decreased to similar negative values; monitoring $\Delta v^{(1)}$ and use of Eq. (1) provided a conventional pressure gauge. Maps such as that in Fig. 2 involving variations in two energy shifts, $\Delta v^{(1)}$ and $\Delta v^{(2)}$, enable representation of raw experimental data over contours of a single stress component of relevance, in this case, the hydrostatic stress in a DAC experiment, $\sigma_{\mathrm{M}}$. Equations (7) and (9) can also be used to calculate contours of other stress components for assessment of other measured experimental trajectories, e.g., $\sigma_{c}$ or $\sigma_{a}$ in intended uniaxial experiments [Fig. 1(b)]. Such assessments can be qualitative, such as the systematic difference between the open and closed symbols in Fig. 2, or quantitative, such as the potential use of $\Delta v^{(2)}$ from Fig. 2 to provide a different estimate of $p$.

Experimental behavior is more conveniently represented, however, by using Eq. (9) to convert the experimental energy shift measurements to $\left(\sigma_{a}, \sigma_{c}\right)$ stress component trajectories as in Fig. 3(a). In this case, the variations in the energy shifts are implicit, and two stress components of relevance are considered; there is no net gain or loss of information. Initially, $\sigma_{c}=\sigma_{a}=0$ in Fig. 3(a). Both stresses decreased to negative values as the experiments proceeded. The closed symbols represent near-hydrostatic conditions in which $\sigma_{c} \approx \sigma_{a}$ (solid diagonal line). The open symbols represent considerable deviation from hydrostatic conditions by spurious superimposition of shear stress such that $\sigma_{c} \approx \sigma_{a} / 2$. Okai et al. suggested that 
simultaneous use of $\Delta v^{(1)}$ and $\Delta v^{(2)}$ could be used to increase the precision of pressure estimation, using both $\Delta v^{(1)}$ and $\Delta v^{(2)}$ in assumed Eq. (1) [35]. Figure 3(a) makes clear that simultaneous use of $\Delta v^{(1)}$ and $\Delta v^{(2)}$ can also be used to increase accuracy by estimation of the actual stress state using Eq. (9). Table 1 summarizes the cited and newly analyzed stress states and maximum stress values from Fig. 3(a).

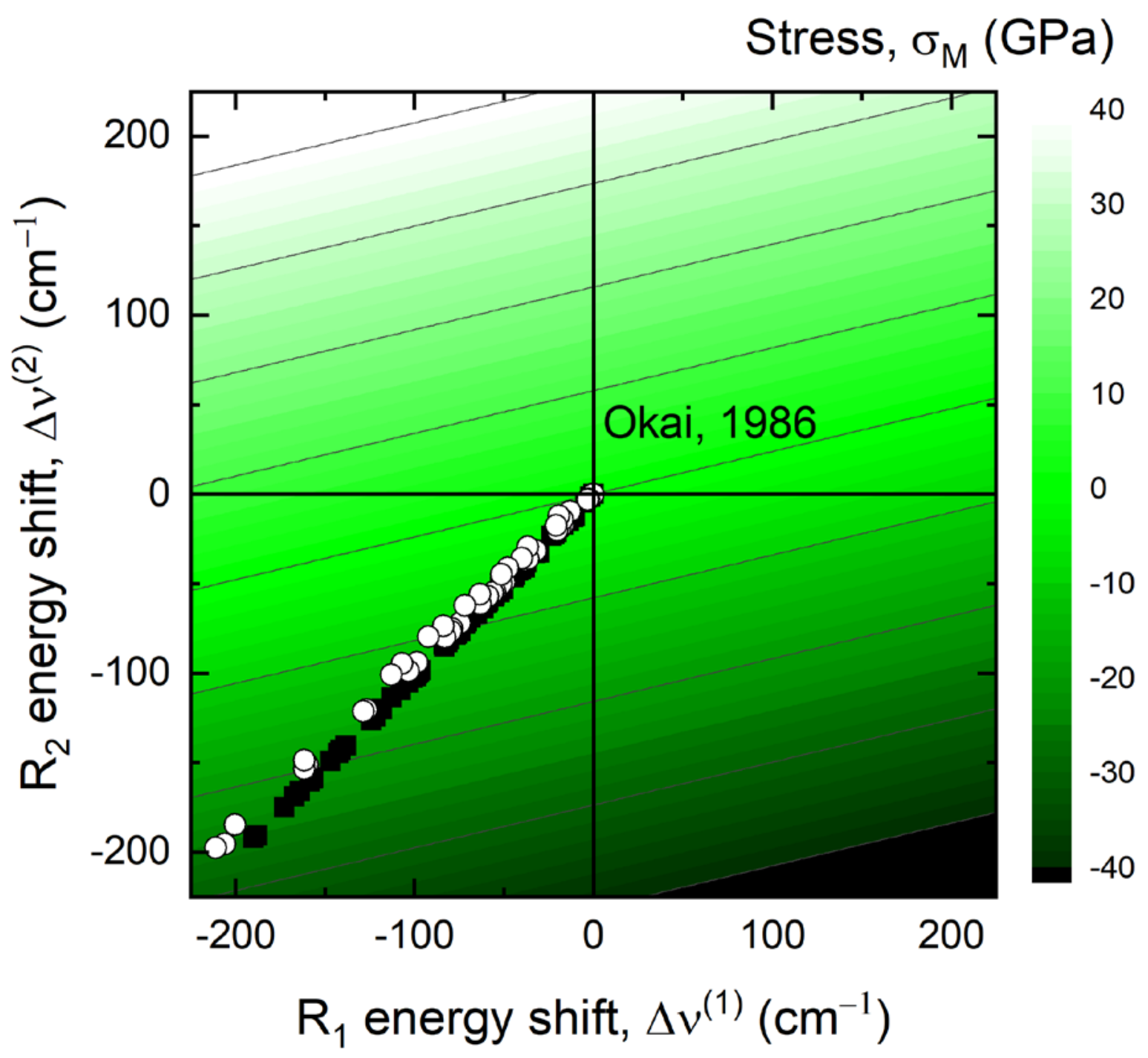

Fig. 2. Color-filled contour plot of mean stress in $\mathrm{Al}_{2} \mathrm{O}_{3}$ as a function of $\mathrm{R}_{1}$ and $\mathrm{R}_{2}$ fluorescence energy shifts. Experimental observations on single crystals of $\mathrm{Al}_{2} \mathrm{O}_{3}$ in high-pressure DACs with different confining media [35] are shown as symbols; closed, near hydrostatic; open, superposed shear. 


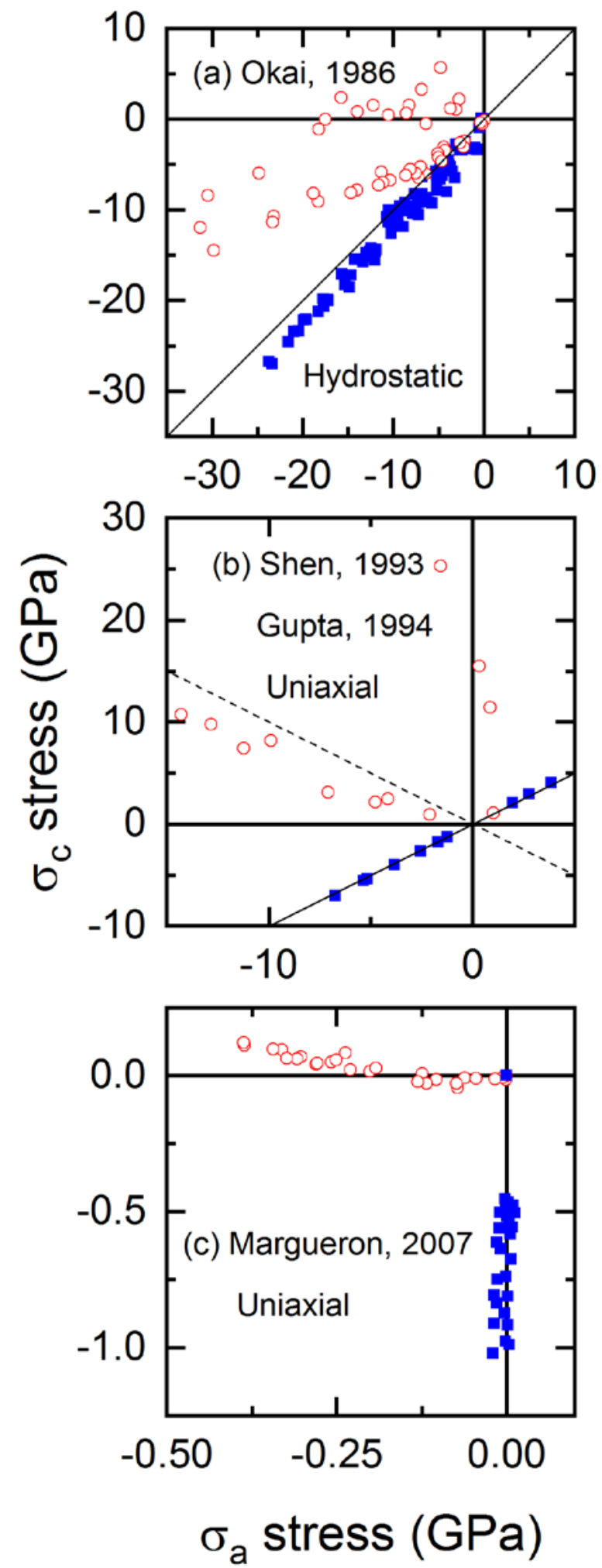

Fig. 3. Crystallographic stress variations determined by new analysis of fluorescence measurements in single crystals of $\mathrm{Al}_{2} \mathrm{O}_{3}$ mechanically loaded in different configurations, with nominal loading indicated. (a) High-pressure DAC [35], open and closed symbols as in Fig. 2. (b) Shock [36, 37]. (c) Quasi-static [38]. Open and closed symbols in (b) and (c) represent nominal uniaxial $\sigma_{a}$ and $\sigma_{\mathrm{c}}$ stress states, respectively. 
Figure 3(b) shows the behavior at somewhat smaller stress values during $a$-axis and $c$-axis oriented single-crystal dynamic shock experiments by Gupta and colleagues [36, 37] [Fig. 1(b)]. Energy shift values for use in Eq. (9) were determined by converting the reported wavelength shift measurements. The $c$-axis results are shown as the closed symbols and exhibit near-equiaxed conditions (solid diagonal line) over both compressive and tensile loading, with $\sigma_{a} \approx \sigma_{c}$, rather than the intended dominant $\sigma_{c}$ states with $\sigma_{a} \approx 0$ (solid vertical line). The $a$-axis results are shown as the open symbols and exhibit near pure shear conditions: In compressive $\sigma_{a}$ loading, $\sigma_{c} \approx-\sigma_{a}$ (dashed diagonal line), rather than the intended dominant $\sigma_{a}$ states with $\sigma_{c} \approx 0$ (solid horizontal line); tensile $\sigma_{a}$ experiments were conducted but resulted in very small $\sigma_{a}$ stress and dominant tensile $\sigma_{c}$ stress (solid vertical line). In both sets of experiments, the variations in $\Delta v^{(1)}$ and $\Delta v^{(2)}$ were monotonic and there was strong correlation between $\Delta v^{(1)}$ and $\Delta v^{(2)}$, leading to the impression that the stress variations were also monotonic, extended over the intended compressive and tensile ranges, and were predominantly uniaxial. Figure 3(b) suggests that the deviations from this impression were significant, and they are summarized in Table 1.

Figure 3(c) shows the behavior at even smaller stress values during $a$-axis and $c$-axis oriented singlecrystal uniaxial quasi-static experiments by Margueron and Clarke [38] [Fig. 1(b)]. Energy shift values for use in Eq. (9) were determined by converting the reported relative energy shifts. The $c$-axis results are shown as the closed symbols and exhibit near-ideal uniaxial conditions (solid vertical line) in compressive loading, with dominant $\sigma_{c}$ states with $\sigma_{a} \approx 0$. The $a$-axis results are shown as the open symbols and exhibit considerable deviation from uniaxial conditions in compressive loading (solid horizontal line) by superimposition of shear stress such that $\sigma_{c} \approx-\sigma_{a} / 4$. Table 1 summarizes the stresses from Fig. 3(c).

Table 1. Stress measurements in $\mathrm{Al}_{2} \mathrm{O}_{3}$ using fluorescence.

\begin{tabular}{|c|c|c|c|c|}
\hline Author & Specimen & Experiment & $\begin{array}{c}\text { Cited Stress } \\
\text { State, Magnitude (GPa) }\end{array}$ & $\begin{array}{c}\text { New Analysis Stress } \\
\text { State, Magnitude (GPa) }\end{array}$ \\
\hline Okai, 1986 [35] & Single crystal & Quasi-static DAC & Hydrostatic, 0 to -22 & Hydrostatic, 0 to -24 \\
\hline Okai, 1986 [35] & Single crystal & Quasi-static DAC & Hydrostatic + shear, 0 to -27 & Hydrostatic + shear, 0 to -32 \\
\hline Shen, 1993 [36] & Single crystal & Shock & $\begin{array}{c}a \text {-axis uniaxial, } \\
-12.5 \text { to } 10.5\end{array}$ & $\begin{array}{c}\text { Shear, transverse uniaxial, } \\
-14.3 \text { to } 1\end{array}$ \\
\hline Gupta, 1994 [37] & Single crystal & Shock & $\begin{array}{l}c \text {-axis uniaxial, } \\
-11 \text { to } 11\end{array}$ & $\begin{array}{l}\text { Hydrostatic, } \\
-7 \text { to } 4\end{array}$ \\
\hline Margueron, 2007 [38] & Single crystal & Quasi-static & $\begin{array}{l}c \text {-axis uniaxial, } \\
\quad \approx-0.5\end{array}$ & $\begin{array}{l}\text { Uniaxial, } \\
-0.4 \text { to }-1\end{array}$ \\
\hline Margueron, 2007 [38] & Single crystal & Quasi-static & $\begin{array}{l}a \text {-axis uniaxial, } \\
\quad \approx-0.5\end{array}$ & $\begin{array}{l}\text { Uniaxial + shear, } \\
\quad 0 \text { to }-0.4\end{array}$ \\
\hline Lipkin, 1996 [39] & Polycrystalline film & $\begin{array}{l}\text { CTE mismatched } \\
\text { substrate }\end{array}$ & Random, -2.5 to -6.7 & Biaxial + shear, -3 \\
\hline Schlichting, 2000 [40] & Polycrystalline film & Substrate & Random, -2 to -1 & Biaxial + shear, -3 \\
\hline Margueron, 2006 [41] & Polycrystalline film & $\begin{array}{l}\text { Substrate + quasi- } \\
\text { static }\end{array}$ & Uniaxial, +0.5 to -7 & Biaxial + shear, 0 to -7 \\
\hline Heeg, 2011 [42] & Polycrystalline film & Substrate & Biaxial, 0 to 1.4 & Biaxial + shear, -3 \\
\hline Nychka, 2001 [43] & Polycrystalline film & Substrate & Random, 0 to -5.5 & Biaxial, 0 to -6 \\
\hline Selcuk, 2002 [44] & Polycrystalline film & Substrate & Random, -1 to -5 & Biaxial, 0 to -5.7 \\
\hline Grabner, 1978 [28] & Bulk polycrystal & CTE anisotropy & Random, 0.065 to 0.260 & Random, 0.10 to 0.45 \\
\hline Michaels, 2018 [32] & Bulk polycrystal & CTE anisotropy & Random, -0.090 to 0.180 & Random, -0.090 to 0.180 \\
\hline
\end{tabular}




\subsection{Polycrystalline Films}

The results above considered stress generation in $\mathrm{Al}_{2} \mathrm{O}_{3}$ single crystals by mechanical loading. Consideration now turns to stress generation in $\mathrm{Al}_{2} \mathrm{O}_{3}$ polycrystals by CTE effects. Polycrystalline $\mathrm{Al}_{2} \mathrm{O}_{3}$ forms as a thermally grown oxide (TGO) film during partial oxidation of metal substrates at elevated temperatures. On cooling, CTE mismatch between film and substrate leads to considerable biaxial stress in the film-usually compressive as the metallic substrate has the greater CTE. An estimate of the film stress is $M \Delta \alpha \Delta T$, where $M$ is a characteristic film elastic modulus, $\Delta \alpha$ is a characteristic film-substrate CTE mismatch, and $\Delta T$ is a characteristic change from the oxidation to the measurement temperature. Using $M=$ $300 \mathrm{GPa}, \Delta \alpha=10^{-5} \mathrm{~K}^{-1}$, and $\Delta T=-1000 \mathrm{~K}[2,4-6]$ gives a characteristic stress of $-3 \mathrm{GPa}$. Figure 4 shows as open symbols the stress values, of about this magnitude, determined for four TGO film-on-substrate systems by Clarke and colleagues and Schlichting et al. [39-42]. The $\sigma_{a}$ stresses are compressive (about -3 $\mathrm{GPa})$ and the $\sigma_{c}$ stress is less but variable ( $\left.< \pm 1 \mathrm{GPa}\right)\left(\sigma_{c}=0\right.$ is shown as the solid horizontal line), implying the textured "mosaic" microstructure shown in the schematic diagram of Fig. 1(c). Within the grains in the $\mathrm{Al}_{2} \mathrm{O}_{3}$ TGO film, the $a$ axes were preferentially parallel to the constraining substrate and the $c$ axes were perpendicular to the substrate and free. This microstructural implication is supported by scanning and transmission electron microscope observations [45, 46] and the extensive mechanical experiments of Nychka and Clarke and Selcuk and Atkinson [43, 44], in which the substrate was varied systematically, leading to the two lower best-fit straight lines in Fig. 4. In both cases, the results were nearly identical, with the $\sigma_{a}$ stresses significantly compressive (up to $-6 \mathrm{GPa}$ ) and covering a range, reflecting the different substrates, and the $\sigma_{c}$ stress less and tensile ( $<1 \mathrm{GPa}$ ) and well correlated, such that $\sigma_{c} \approx-\sigma_{a} / 6$. (For clarity, the stress uncertainties, about $\pm 1 \mathrm{GPa}$, derived from the energy shift uncertainties $[43,44]$ are not shown.) In systematic mechanical loading experiments by Margueron and Clarke on microscale beams formed from TGO films [41], mechanical stresses were superimposed on the CTE mismatch stress by bending. The results of these experiments are shown as the upper curved line in Fig. 4, extending in both directions from the open symbol representing the as-deposited unloaded configuration. The mechanical loading generated near pure shear stress conditions with $\sigma_{c} \approx-\sigma_{a}$ over substantial compressive $\sigma_{a}$ and tensile $\sigma_{c}$ stresses rather than the intended dominant uniaxial stress. The intended compressive and tensile $\sigma_{a}$ stresses were not achieved, and the system exhibited clear nonlinearity for $\sigma_{a} \approx 0$ (the stresses were very large, and the nonlinearity may have arisen from inelastic deformation). Energy shift values for use in Eq. (9) to calculate the stress values in Fig. 4 were determined from reported fluorescence spectra or graphical or numerical descriptions as points or lines of absolute or relative energy shifts. Table 1 summarizes the stresses from Fig. 4.

\subsection{Bulk Polycrystals}

CTE anisotropy of corundum grains leads to general triaxial residual stress distributions in dense polycrystalline $\mathrm{Al}_{2} \mathrm{O}_{3}$. An estimate of the residual stresses is still $M \Delta \alpha \Delta T$ as above, but in this case $\Delta \alpha$ is a characteristic CTE difference between differently oriented corundum grains within the polycrystalline $\mathrm{Al}_{2} \mathrm{O}_{3}$. Using $M=300 \mathrm{GPa}$ and $\Delta T=-1000 \mathrm{~K}$ as before, but now $\Delta \alpha= \pm 10^{-6} \mathrm{~K}^{-1}$ [5] gives characteristic stresses of $\pm 300 \mathrm{MPa}$. The earliest measurements of fluorescence energy shifts in polycrystalline $\mathrm{Al}_{2} \mathrm{O}_{3}$, shown in the schematic diagram of Fig. 1(d), revealed the effects of residual stresses [28] of this magnitude. The reported shifts were analyzed using Eq. (9), and the resultant global stresses for three polycrystalline $\mathrm{Al}_{2} \mathrm{O}_{3}$ materials are shown as symbols in Fig. 5(a). The solid squares represent the mean stress values determined from the reported mean energy shifts. The bars represent the widths of the stress distributions and were determined from the reported widths in the energy shift spectra (the bars are not stress uncertainties) [32]. The data in Fig. 5(a) are representative of an "integrated scan," in which a single large probe simultaneously illuminates many polycrystalline grains [Fig. 1(d)]. As observed in the recent studies, [24, 25, 30-33] and reflecting the well-known greater CTE along the $c$ axis relative to the $a$ axes $[1-3,8-10,13,14]$, the mean $\sigma_{c}$ residual stress is tensile and the mean $\sigma_{a}$ residual stress is compressive, with characteristic magnitudes of hundreds of megapascals. Further, the global mean values are consistent with the equilibrium condition of $\sigma_{c}=-2 \sigma_{a}$ (shown as the dashed diagonal line). The dispersions in the stress distributions are substantial, also hundreds of megapascals, reflecting the random misorientations of 
adjacent grains in the polycrystalline microstructures. The most recent measurements of the globally averaged stresses in a series of polycrystalline $\mathrm{Al}_{2} \mathrm{O}_{3}$ materials [32] considerably reduced the scatter and uncertainty in graphs such as Fig. 5(a) but did not alter the conclusions regarding the signs, magnitudes, or equilibrium of the mean stresses. However, the mapping approach of the recent work, in which local properties were probed, shown in the schematic diagram of Fig. 1(e), greatly increased the statistical information available regarding the distributions of stresses. An example is shown in Fig. 5(b), which is a scatter plot of the $>16000 \sigma_{c}$ vs. $\sigma_{a}$ observations from a single map of a single polycrystalline $\mathrm{Al}_{2} \mathrm{O}_{3}$. The data in Fig. 5(b) are representative of a mapping approach, in which multiple small probed areas are illuminated [Fig. 1(e)]. The extent and density of the data in Fig. 5(b) reflect the range of stress states in the material and illustrate that the signs, magnitudes, and equilibrium states of the stresses only agree with average expectations; there is considerable dispersion about the average values, reflecting local microstructural effects. In particular, the dashed line represents the equilibrium conditions of $\sigma_{c}=-2 \sigma_{a}$ or $\sigma_{\mathrm{M}}=0$. Points above this line thus represent net tension locations in the microstructure with mean stress $\sigma_{\mathrm{M}}$ $>0$, and points below the line represent net compression locations with $\sigma_{\mathrm{M}}<0$. Extremes of these locations exhibit compressive stress $\sigma_{c}<0$ (below horizontal line) and tensile stress $\sigma_{a}>0$ (right of vertical line).

Microstructural effects, probably grain scale related, are also apparent in the clusters within the data. Nevertheless, it is clear that the dispersion of individual measurements observed explicitly in the recent measurements [32] [see Fig. 5(b)] is reflected in the widths of the bars in the re-analyzed early measurements [28] [see Fig. 5(a)].

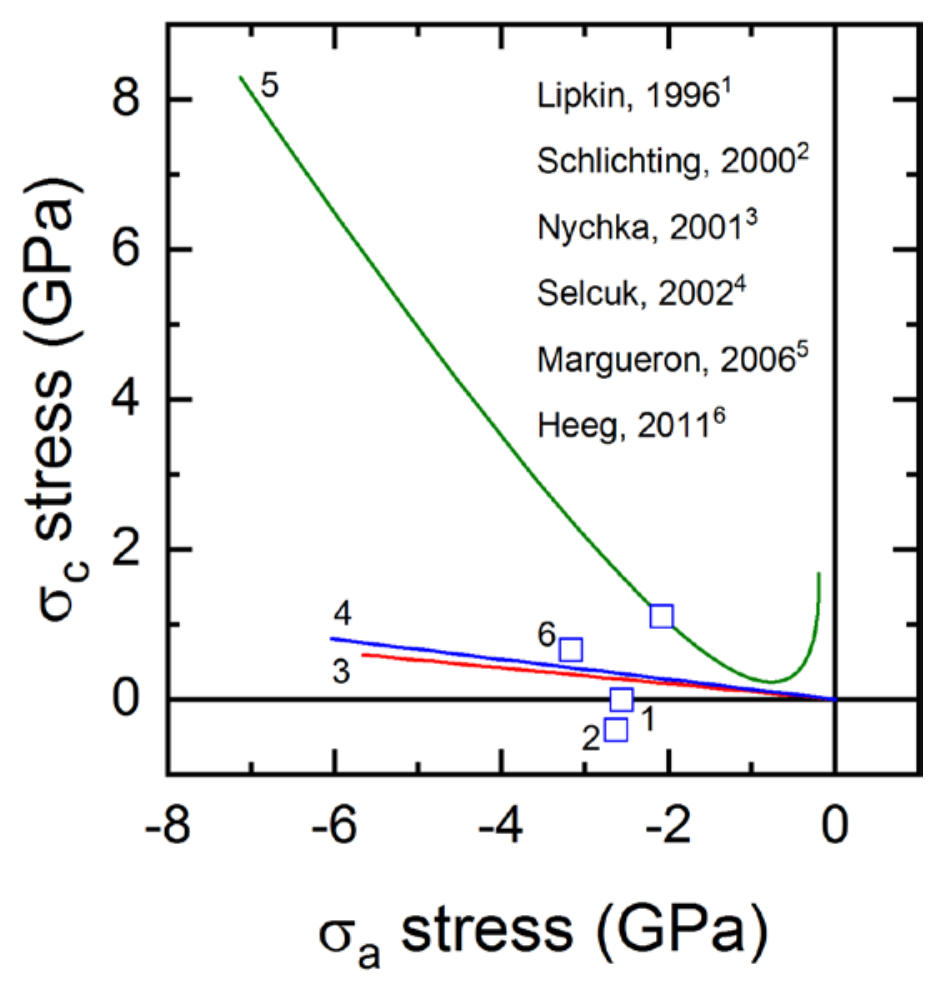

Fig. 4. Crystallographic stress variations determined by new analysis of fluorescence measurements in polycrystalline films of $\mathrm{Al}_{2} \mathrm{O}_{3}$ mechanically loaded by CTE mismatched substrates. [39-44] Symbols $(1,2,6)$ and lines (3, 4): as-deposited films. Line (5): film additionally mechanically loaded in bending. 


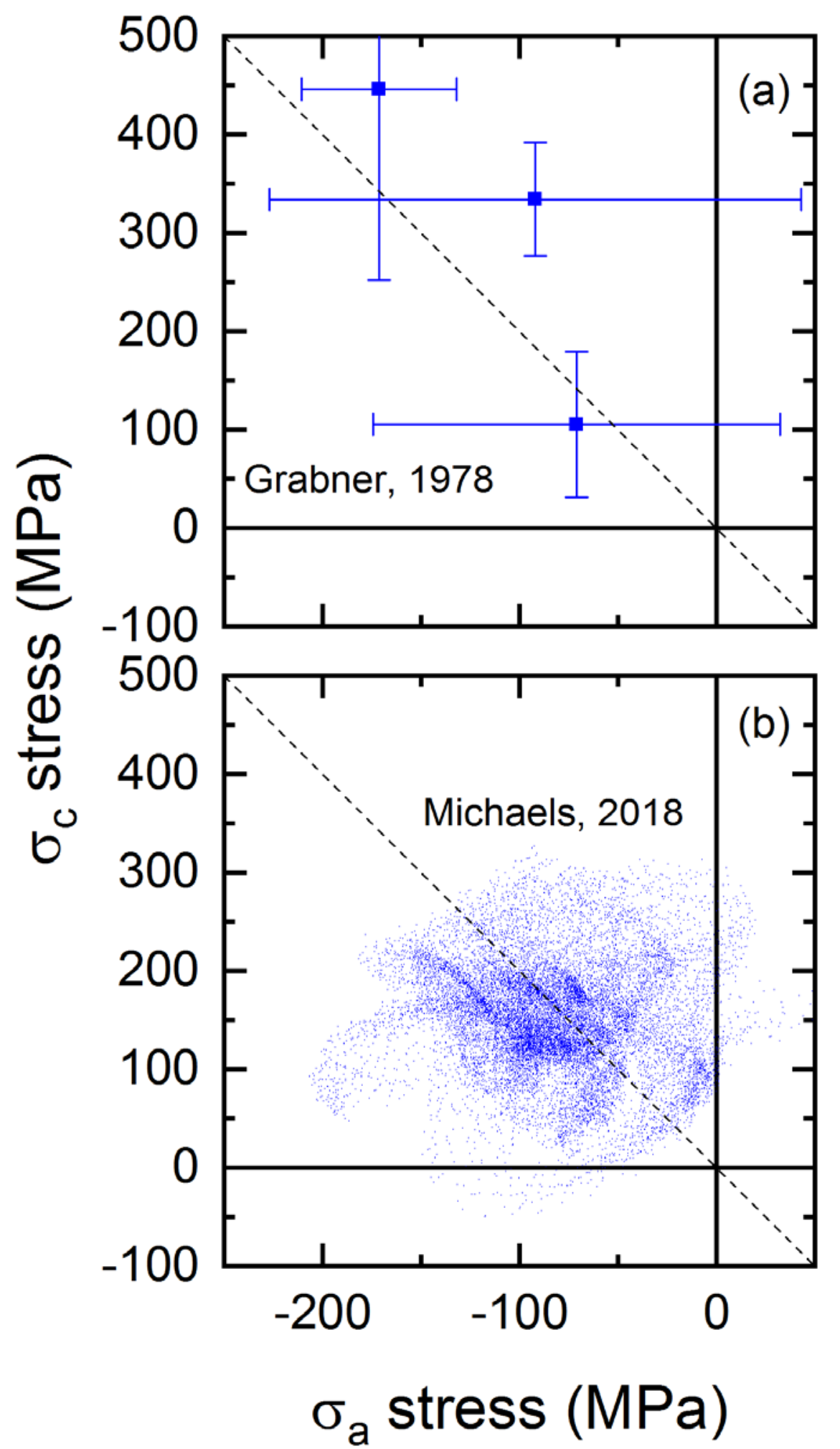

Fig. 5. Crystallographic stress variations determined by new analysis of fluorescence measurements in bulk polycrystalline $\mathrm{Al}_{2} \mathrm{O}_{3}$ containing residual stress due to anisotropic CTE. (a) Integrated scan measurements [28]. (b) Two-dimensional mapping measurements [32]. 


\section{Discussion}

In all cases of revisiting and reanalyzing the above fluorescence measurements, the recent two-peak analysis method [Eqs. (1) to (9)] provided new, calibrated, stress information that was previously unavailable or erroneously calculated using previous single-peak methods. The additional information placed prior assumptions into three categories: (1) In some cases, revisiting the measurements confirmed prior assumptions, such as the near-hydrostatic pressure states in DAC experiments, as in Fig. 3(a) [35], suggested by the absence of peak shift splitting, or the near-uniaxial $\sigma_{c}$ stress states in quasi-static experiments, as in Fig. 3(c) [38], set by test configuration. (2) In other cases, revisiting the measurements led to results, unknown earlier, but that are in accord with more recent ideas, such as the nonhydrostatic stress states in DAC experiments, as in Fig. 3(a) [35], suggested by peak shift splitting, or the equilibrium residual stress configuration and dispersion in the early polycrystalline material experiments, as in Fig. 5(a) [28]. (3) A clear conclusion in some cases, however, is that the intended or assumed experimental stress states were not achieved, such as the hydrostatic or shear configurations generated in the intended uniaxial shock experiments, as in Fig. 3(b) [36, 37], or the shear configurations generated in the assumed uniaxially stressed $\sigma_{a}$ single crystal in Fig. 3(c) [38] or TGO film in Fig. 4 [41].

The differences between the assumed and actual stress states in this last case (category 3 ) have two important consequences. First, sophisticated atomic-scale models have been developed to relate fluorescence energy shift to the stress or, importantly, strain state that were instigated by the shock experiments [47] and refined and calibrated by quasi-static experiments [48]. The analysis here suggests that validation and application of the models probably rests on false assumptions regarding stress states. The usefulness of such models is to enable fluorescence energy shift measurements, perhaps combined with separate intensity [30, 32], orientation [31, 33], or polarization [38] measurements, to determine complex stress states, perhaps the entire stress tensor, beyond the two components estimated here. Applications might include stress states in deliberately nonhydrostatic environments [49], or adjacent to inclusions in geological structures [50]. Second, larger-scale materials science-based models of equilibrium stress distributions, e.g., Ref. [11], or kinetically limited stress development, e.g., Ref. [2], in polycrystalline $\mathrm{Al}_{2} \mathrm{O}_{3}$ fabrication have been developed that are at least partially based on evidence from fluorescence measurements [19]. The analysis here suggests that the many such models for residual stress in $\mathrm{Al}_{2} \mathrm{O}_{3}$ and other materials [1-3, 7-14] may lack firm calibration data. The importance of these microstructure-scale models is to enable design of materials and prediction of damage, strength, and reliability [15, 17, 24].

Finally, the TGO results in Fig. 4 generated by revisiting earlier measurements [39-44] provide new information and new questions. The as-fabricated compressive $\sigma_{a}$ stresses in Fig. 4 are easily understood as deriving from CTE mismatch with the underlying metal substrates. However, if the TGO microstructures consisted of randomly oriented polycrystals similar to those in Fig. 1(d) and (e), the $c$-axis constraint and substrate CTE mismatch would be similar to the $a$ axis, and the $\sigma_{c}$ stresses would also be compressive and of similar magnitude to $\sigma_{a}$. This is not observed. Alternatively, if the TGO microstructures were aligned mosaic polycrystals as observed $[45,46]$ and depicted in Fig. 1(c), the $c$ axes would be unconstrained, and the $\sigma_{c}$ stresses would be near zero for all substrates. This stress state is also not observed. For either microstructure, the relationship between the stress components should be unchanged by mechanical loading, as in Ref. [29] and not, as observed [41], develop significant tension. A potential explanation for the results of Fig. 4 is that the fluorescence energy shifts reflect the state of strain, not stress, in the material as follows: On TGO development, the mosaic structure forms as observed and negative $a$-axis strain develops due to CTE mismatch that is controlled by substrate selection and mechanical loading. Due to Poisson effects, the traction-free $c$ axis develops a positive strain in response. A strain-based model [47], which is somewhat similar to the approach used in the recent calibration [48], could thus explain the observations in an important application and extend to other cases (e.g., Figs. 2, 3, and 5). Although a strain-based fluorescence energy shift analysis might be more easily applied in cases in which deformation is specified (as in Fig. 4), such a model would be one step removed from the specification of stresses, which are usually required in fracture models $[15,17]$. 


\section{Conclusions}

Previous applications of single-peak-based fluorescence methods in single-crystal and polycrystalline alumina have in many cases led to incorrect qualitative and quantitative inferences regarding applied or microstructurally driven residual stress states. A newly developed method, based on simultaneous measurement of energy shifts in both the $\mathrm{R}_{1}$ and $\mathrm{R}_{2}$ peaks, shows that many previously assumed uniaxial stress states contained significant shear components or were of opposite sign to those assumed. In many cases, the new method suggests values for stress that differ by factors of two or more from those originally inferred. In addition, the new findings suggest that microstructures in TGO films do not consist of randomly oriented grains and that stress states in some early shock experiments are not a reliable platform for development of atomistic models of fluorescence shift with stress. Revisiting fluorescence shifts in polycrystalline alumina showed that the earliest measurements are consistent with stress dispersion and equilibrium conditions revealed in more recent and statistically complete measurements.

\section{Acknowledgments}

The authors thank Drs. Y. B. Gerbig, D. L. Kaiser, and J. E. Maslar, all of the National Institute of Standards and Technology (NIST), and Dr. G. A. Myers, of Welldog, for careful review of the manuscript. RFC is pleased to have known NIST researchers Drs J. B. Wachtman Jr. and G. J. Piermarini.

\section{References}

[1] Blendell JE, Coble RL, Charles RJ (1976) On the relaxation of stresses arising from thermal expansion anisotropy during cooling polycrystalline materials. Ceramic Microstructures, ed Fulrath RM, Pask JA (Westview Press, Boulder, CA), pp 721730 .

[2] Blendell J, Coble R (1982) Measurement of stress due to thermal expansion anisotropy in $\mathrm{Al}_{2} \mathrm{O}_{3}$. Journal of the American Ceramic Society 65(3):174-178. https://doi.org/10.1111/j.1151-2916.1982.tb10390.x

[3] Evans AG (1978) Microfracture from thermal expansion anisotropy-I. Single phase systems. Acta Metallurgica 26(12):18451853. https://doi.org/10.1016/0001-6160(78)90097-4

[4] Wachtman JB Jr, Tefft WE, Lam DG Jr, Stinchfield RP (1960) Elastic constants of synthetic single crystal corundum at room temperature. Journal of Research of the National. Bureau of Standards-A. Physics and Chemistry 64A(3):213-228. https://doi.org/10.1111/j.1151-2916.1960.tb13663.x

[5] Wachtman JB Jr, Scuderi TG, Cleek GW (1962) Linear thermal expansion of aluminum oxide and thorium oxide from $100^{\circ}$ to $1100^{\circ}$ K. Journal of the American Ceramic Society 45(7):319-323. https://doi.org/10.1111/j.1151-2916.1962.tb11159.x

[6] Munro RG (1997) Evaluated material properties for a sintered $\alpha$-alumina. Journal of the American Ceramic Society 80(8):19191928. https://doi.org/10.1111/j.1151-2916.1997.tb03074.x

[7] Clarke FJP (1964) Residual strain and the fracture stress-grain size relationship in brittle solids. Acta Metallurgica 12(2):139143. https://doi.org/10.1016/0001-6160(64)90181-6

[8] Evans AG, Clarke DR (1980) Residual stresses and microcracking induced by thermal contraction inhomogeneity. Thermal Stresses in Materials and Structures, ed Hasselmann DPH, Heller RA (Plenum Press, New York), pp 629-648. https://doi.org/10.1007/978-1-4613-3156-8_39

[9] Clarke DR (1980) Microfracture in brittle solids resulting from anisotropic shape changes. Acta Metallurgica 28(7):913-924. https://doi.org/10.1016/0001-6160(80)90107-8

[10] Tvergaard V, Hutchinson JW (1988) Microcracking in ceramics induced by thermal expansion or elastic anisotropy. Journal of the American Ceramic Society 71(3):157-166. https://doi.org/10.1111/j.1151-2916.1988.tb05022.x

[11] Kreher W, Molinari A (1993) Residual stresses in polycrystals as influenced by grain shape and texture. Journal of the Mechanics and Physics of Solids 41(12):1955-1977. https://doi.org/10.1016/0022-5096(93)90075-Q

[12] Ortiz M, Suresh S (1993) Statistical properties of residual stresses and intergranular fracture in ceramic materials. Journal of Applied Mechanics 60(1):77-84. https://dx.doi.org/10.1115/1.2900782

[13] Zimmermann A, Fuller ER Jr, Rödel J (1999) Residual stress distributions in ceramics. Journal of the American Ceramic Society 82(11):3155-3160. https://doi.org/10.1111/j.1151-2916.1999.tb02217.x

[14] Vedula VR, Glass SJ, Saylor DM, Rohrer GS, Carter WC, Langer SA, Fuller ER Jr (2001) Residual-stress predictions in polycrystalline alumina. Journal of the American Ceramic Society 84(12):2947-2954. https://doi.org/10.1111/j.11512916.2001.tb01119.x

[15] Bennison SJ, Lawn BR (1989) Role of interfacial grain-bridging sliding friction in the crack-resistance and strength properties of nontransforming ceramics. Acta Metallurgica 37(10):2659-2671. https://doi.org/10.1016/0001-6160(89)90299-X

[16] Lawn BR (1991) Fundamental condition for the existence of microcrack clouds in monophase ceramics. Journal of the European Ceramic Society 7:17-20. https://doi.org/10.1016/0955-2219(91)90048-5

[17] Galal Yousef S, Rödel J, Fuller ER Jr, Zimmermann A, El-Dasher BS (2005) Microcrack evolution in alumina ceramics: Experiment and simulation. Journal of the American Ceramic Society 88(10):2809-2816. https://doi.org/10.1111/j.15512916.2005.00312.x 
[18] Aswad MA, Marrow TJ (2012) Intergranular crack nuclei in polycrystalline alumina. Engineering Fracture Mechanics 95(SI):29-36. https://doi.org/10.1016/j.engfracmech.2012.08.005

[19] Ma Q, Clarke DR (1994) Piezospectroscopic determination of residual stresses in polycrystalline alumina, Journal of the American Ceramic Society 77(2):298-302. https://doi.org/10.1111/j.1151-2916.1994.tb06996.x

[20] Ma Q, Pompe W, French JD, Clarke DR (1994) Residual stresses in $\mathrm{Al}_{2} \mathrm{O}_{3}-\mathrm{ZrO}_{2}$ composites: A test of stochastic stress models. Acta Metallurgica Materialia 42(5):1673-1681. https://doi.org/10.1016/0956-7151(94)90377-8

[21] Sergo V, Pezzotti G, Sbaizero O, Nishida T (1998) Grain size influence on residual stresses in alumina/zirconia composites. Acta Materialia 46(5):1701-1710. https://doi.org/10.1016/S1359-6454(97)00348-0

[22] Messaoudi K, Huntz AM, Di Menza L (2000) Residual stresses in alumina scales. Experiments, modeling, and stress-relaxation phenomena. Oxidation of Metals 53(1/2):49-75. https://doi.org/10.1023/A:1004530729859

[23] Gonzalez D, King A, Mostafavi M, Reischig P, Rolland du Roscoat S, Ludwig W, Quinta da Fonseca J, Withers PJ, Marrow TJ (2013) Three-dimensional observation and image-based modelling of thermal strains in polycrystalline alumina. Acta Materialia 61(20):7521-7533. https://doi.org/10.1016/j.actamat.2013.06.005

[24] Michaels CA, Cook RF (2016) Determination of residual stress distributions in polycrystalline alumina using fluorescence microscopy. Materials and Design 107:478-490. https://doi.org/10.1016/j.matdes.2016.06.063

[25] Cook RF, Michaels CA (2017) Review: Coefficients for stress, temperature, and composition effects in fluorescence measurements of alumina. Journal of Research of the National Institute of Standards and Technology 122:43. https://doi.org/10.6028/jres.122.043

[26] Forman R, Piermarini G, Barnett J, Block S (1972) Pressure measurement made by the utilization of ruby sharp-line luminescence. Science 176(4032):284-285. https://doi.org/10.1126/science.176.4032.284

[27] Syassen K (2008) Ruby under pressure. High Pressure Research 28(2):75-126. https://doi.org/10.1080/08957950802235640

[28] Grabner L (1978) Spectroscopic technique for the measurement of residual stress in sintered $\mathrm{Al}_{2} \mathrm{O}_{3}$. Journal of Applied Physics 49(2):580-583. https://doi.org/10.1063/1.324682

[29] Ma Q, Clarke DR (1993) Stress measurement in single-crystal and polycrystalline ceramics using their optical fluorescence. Journal of the American Ceramic Society 76(6):1433-1440. https://doi.org/10.1111/j.1151-2916.1993.tb03922.x

[30] Myers GA, Michaels CA, Cook RF (2016) Quantitative mapping of stress heterogeneity in polycrystalline alumina using hyperspectral fluorescence microscopy. Acta Materialia 106:272-282. https://doi.org/10.1016/j.actamat.2016.01.020

[31] Teague MC, Rodgers T, Grutzik S, Meserole S (2018) Characterization and modeling of microstructural stresses in alumina. Journal of the American Ceramic Society 101(5):2155-2161. https://doi.org/10.1111/jace.15369

[32] Michaels CA, Cook RF (2018) Residual stress in polycrystalline alumina: Comparison of two-dimensional maps and integrated scans in fluorescence-based measurements. Acta Materialia 159:309-319. https://doi.org/10.1016/j.actamat.2018.08.025

[33] Grutzik SJ, Teague MC (2019) Full stress tensor measurement using fluorescence spectroscopy. Journal of Applied Physics 125:155105. https://doi.org/10.1063/1.5088584

[34] Dauskardt RH, Ager JW III (1996) Quantitative stress mapping in alumina composites by optical fluorescence imaging. Acta Materialia 44(2):625-641. https://doi.org/10.1016/1359-6454(95)00189-1

[35] Okai B, Shimomura O, Fujishiro I (1986) Measurement of anisotropic stress field in a gasketed diamond-anvil cell. Physica B\&C 139(1-3):799-802. https://doi.org/10.1016/0378-4363(86)90705-9

[36] Shen XA, Gupta YM (1993) Effect of crystal orientation on ruby R-line shifts under shock compression and tension. Physical Review B 48(5):2929-2940. https://doi.org/10.1103/PhysRevB.48.2929

[37] Gupta YM, Horn PD, Burt JA (1994) Effect of tension on R lines in ruby crystals shocked along crystal c axis. Journal of Applied Physics 76(3):1784-1788. https://doi.org/10.1063/1.357696

[38] Margueron SH, Clarke DR (2007) Effect of polarization and uniaxial stress on the R-line luminescence of single crystal sapphire. Journal of Applied Physics 101:094902. https://doi.org/10.1063/1.2717155

[39] Lipkin DM, Clarke DR (1996) Measurement of the stress in oxide scales formed by oxidation of alumina-forming alloys. Oxidation of Metals 45(3-4):267-279. https://doi.org/10.1007/BF01046985

[40] Schlichting KW, Vaidyanathan K, Sohn YH, Jordan EH, Gell M, Padture N (2000) Application of $\mathrm{Cr}^{3+}$ photoluminescence piezo-spectroscopy to plasma-sprayed thermal barrier coatings for residual stress measurement. Materials Science and Engineering A 291(1-2):68-77. https://doi.org/10.1016/S0921-5093(00)00973-4

[41] Margueron SH, Clarke DR (2006) The use of polarization in the piezospectroscopic determination of the residual stresses in polycrystalline alumina films. Acta Materialia 54(20):5551-5557. https://doi.org/10.1016/j.actamat.2006.08.017

[42] Heeg B, Tolpygo VK, Clarke DR (2011) Damage evolution in thermal barrier coatings with thermal cycling. Journal of the American Ceramic Society 94(S1):S112-S119. https://doi.org/10.1111/j.1551-2916.2011.04496.x

[43] Nychka JA, Clarke DR (2001) Damage quantification in TBCs by photo-stimulated luminescence spectroscopy. Surface \& Coatings Technology 146-147:110-116. https://doi.org/10.1016/S0257-8972(01)01455-4

[44] Selcuk A, Atkinson A (2002) Analysis of the $\mathrm{Cr}^{3+}$ luminescence spectra from thermally grown oxide in thermal barrier coatings. Materials Science and Engineering A 355(1-2):147-156. https://doi.org/10.1016/S0921-5093(01)01911-6

[45] Karadge M, Zhao X, Preuss M, Xiao P (2006) Microtexture of the thermally grown alumina in commercial thermal barrier coatings. Scripta Materialia 54(4):639-644. https://doi.org/10.1016/j.scriptamat.2005.10.043

[46] Bai M, Sarakinou E, Chen Y, Daad Chandio A, Zhao X, Preuss M, Xiao P (2015) Microtexture analysis of the alumina scale in thermal barrier coatings. Journal of the American Ceramic Society 98(12):3639-3642. https://doi.org/10.1111/jace.13945

[47] Sharma SM, Gupta YM (1991) Theoretical analysis of R-line shifts of ruby subjected to different deformation conditions. Physical Review B 43(1):879-893. https://doi.org/10.1103/PhysRevB.43.879

[48] Margueron S, Clarke DR (2015) Refined crystal field model for the piezospectroscopy analysis of stresses in polycrystalline alumina. Journal of Applied Physics 118(9):094503. https://doi.org/10.1063/1.4929960

[49] Chai M, Brown JM (1996) Effects of static non-hydrostatic stress on the R lines of ruby single crystals. Geophysical Research Letters 23(24):3539-3542. https://doi.org/10.1029/96GL03372 
[50] Noguchi N, Abduriyim A, Shimizu I, Kamegata N, Odaka S, Kagi H (2013) Imaging of internal stress around a mineral inclusion in a sapphire crystal: Application of micro-Raman and photoluminescence spectroscopy. Journal of Raman Spectroscopy 44(1):147-154. https://doi.org/10.1002/jrs.4161

About the authors: Dr. Robert F. Cook is a NIST Fellow in the Materials Measurement Science Division at NIST. Dr. Cook performs research on mechanics and mechanical behavior of materials, with a focus on fracture and deformation of brittle materials.

Dr. Chris A. Michaels is a research chemist in the Materials Measurement Science Division at NIST. Dr. Michaels develops and applies high-spatial-resolution spectroscopic techniques and instrumentation to the study of materials.

The National Institute of Standards and Technology is an agency of the U.S. Department of Commerce. 